Chapter 9

\title{
Graphene against Other Two-Dimensional Materials: A Comparative Study on the Basis of Photonic Applications
}

\author{
Rafael Vargas-Bernal
}

Additional information is available at the end of the chapter

http://dx.doi.org/10.5772/67807

\begin{abstract}
Two-dimensional materials represent the basis of technological development to produce applications with high added value for nanoelectronics, photonics, and optoelectronics. In first decades of this century, these materials are impelling this development through materials based on carbon, silicon, germanium, tin, phosphorus, arsenic, antimony, and boron. 2D materials for photonic applications used until now are graphene, silicene, germanene, stanene, phosphorene, arsenene, antimonene, and borophene. In this work, the main strategies to modify optical properties of 2D materials are studied for achieving photodetection, transportation, and emitting of light. Optical properties analyzed here are refractive index, extinction coefficient, relative permittivity, absorption coefficient, chromatic dispersion, group index, and transmittance. The transmittance spectra of various two-dimensional materials are presented here with the aim of classifying them from photonic point-of-view. A performance comparison between graphene and other twodimensional materials is done to help the designer choose the best design material for photonic applications. In next three decades, a lot of scientific research will be realized to completely exploit the use of 2D materials either as single monolayers or as stacked multilayers in several fields of knowledge with a special emphasis in the optoelectronics and photonic industry in benefit of the industry and ultimately to our society.
\end{abstract}

Keywords: graphene, 2D materials, photonic devices, optical properties, nanophotonics, optoelectronics 


\section{Introduction}

The urgent need for photonic materials with useful and fascinating properties is leading researchers around the world to exploit technologically Van der Waals materials.Two-dimensional (2D) materials due to their unique optical and electronic properties have an enormous potential in photonics and optoelectronics. These two-dimensional materials are stable, simple, and consisting of layers of few-atom-thick. Particularly, materials such as graphene and its derivatives, two-dimensional materials based on silicon, germanium, tin, phosphorus, arsenic, antimony, bismuth, and boron, as well as transition metal dichalcogenides are being synthesized for the development of innovative applications in the ultraviolet, visible, and infrared and terahertz frequency ranges.

Optoelectronic devices can be defined as electronic devices capable of generating, detecting, interacting with, or controlling light [1,2]. Main applications of flexible electronics that can be impelled using two-dimensional materials are large arrays of solar cells, wearable electronics, and transparent displays. The presence of a direct band gap in two-dimensional monolayer materials makes them completely attractive for optoelectronic applications. Physical properties of two-dimensional materials that are attractive for the development of photonic applications are mechanical flexibility, electrical conductivity, and optical transparency. Solid-state properties such as morphology and crystallinity directly influence the optical properties of two-dimensional materials. 2D materials have the potential to impact technologies of modulation, transmission, and detection of photonic signals [3-5]. These materials are attractive for photonics and optoelectronic devices, such as displays, touch screens, photodetectors, light-emitting diodes (LEDs), and solar cells. Two-dimensional transition metal dichalcogenides (TMDCs) provide higher optical efficiency than their bulk counterparts due to that these nanomaterials present a direct band gap, which is not found in the volumetric versions [6]. Heterostructures based on TDMCs and other materials such as graphene, $h$-BN, and phosphorene present a band gap that can cover a wide spectral range from visible to mid-IR. Integrated photonic systems based on 2D materials can improve network performance while reducing costs.

Despite all the progress made by researchers so far in the development of two-dimensional materials, it has not been possible to understand all conditions that can be used to tune the optical properties, which influence the development of photonic devices and systems. This work has as aim studying the optical properties of two-dimensional materials for the development of applications in photonics and optoelectronics. The transmittance spectra of various two-dimensional materials are presented here with the aim of classifying these in the different types from photonic point-of-view. A performance comparison between graphene and other two-dimensional materials is done to help to the designer to choose the best design material for photonic applications. This document can be considered as a technical resource for academics, researchers, and engineers working in the photonics and optoelectronics and developing novel technologies based on emerging materials. Additionally, the document provides an overview of the state-of-the-art of the photonic applications based on graphene and other two-dimensional materials. A direct comparison of the performance and qualities that different types of two-dimensional nanomaterials have in the photonic applications is made 
with the aim of determining the advantages and disadvantages that the designer can exploit technologically in each of these materials. This chapter has been divided as follows: Section 2 introduces basic concepts about the optical properties that can be found in two-dimensional materials and a comparison of optical performance between graphene and other materials is performed. Main applications of two-dimensional materials in optoelectronics and photonics are described in Section 3. Finally, conclusions about of the work are given in Section 4.

\section{Optical properties of two-dimensional materials}

Light is an electromagnetic radiation within a certain portion or a set of wavelengths of the electromagnetic spectrum. All types of light are emitted, transported, and absorbed in tiny packets called photons and exhibit properties of both wave and particle. The study of light and the interaction of light and matter are known as optics, and it continues being an important research area in modern physics. With the introduction of two-dimensional materials and the control of their optical properties was possible to extend the development of photonic applications to new and novel technological applications of high added value [2].

Main optical properties that allow describing the performance of an engineering material are the following: (1) refractive index, (2) extinction coefficient, (3) relative permittivity, (4) absorption coefficient, (5) chromatic dispersion, and (6) group index. To understand what role each of these optical properties has in the performance of optical materials, the subsequent paragraphs will describe each of them.

Reflection is the change in direction of the beam of light at an interface (boundary between different substances) between two different media so that the wave returns into the medium from which it was generated. This effect can be either specular (mirror-like) or diffuse (retaining the energy, but losing the image) depending on the nature of the interface, as visualized in Figure 1. Incident light (illustrated in red) travels in the medium 1 and strikes against a surface, a fraction of the light is reflected in the same medium (shown in blue), and other

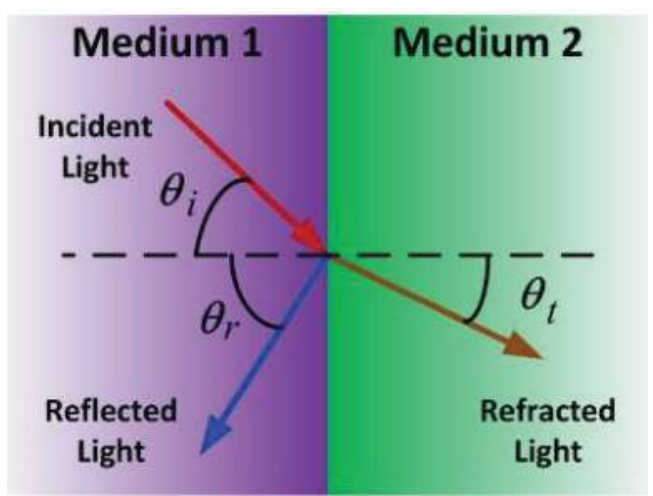

Figure 1. Basic optical phenomena between a pair of physical media. 
fraction of the light is transmitted into or refracted into medium 2 (depicted in brown). When light reflects off a material denser than the external medium, it undergoes a polarity inversion (change of direction). In contrast, a less dense, lower refractive index material will refract or transmit the incident light that was applied to it.

Light propagation in absorbing materials is described using a complex-valued refractive index. The imaginary part is related with the attenuation, while the real part accounts for refraction. The refractive index or index of refraction $n$ of a material can be defined as a dimensionless number that describes how light propagates through that medium. This property represents the rate to which the speed of light in vacuum travels through a specific medium. In other words, it determines how much light is refracted when it enters a material. The refraction is presented by the change in direction of propagation of an electromagnetic wave, oscillation of electrical and magnetic fields with varying or fixed wavelength, due to a change in its transmission medium. The wavelength $(\lambda)$ is the spatial period of a wave, that is, the distance over which the wave's shape repeats, and thus the inverse of the spatial frequency. A transmission medium is a solid material that can propagate light either infrared, visible and/or ultraviolet. In addition, refractive index varies with respect to the wavelength and/or photon energy of the light applied to the material. Visible light is usually defined as that having wavelengths in the range of $400-700 \mathrm{~nm}(430-750 \mathrm{THz})$, and it is between the infrared (with longer wavelengths) and the ultraviolet (with shorter wavelengths). Infrared light is an invisible radiant energy with wavelengths between $700 \mathrm{~nm}(430 \mathrm{THz})$ and 1,000,000 nm (300 GHz). And, ultraviolet light is an electromagnetic radiation with a wavelength from $10 \mathrm{~nm}(30 \mathrm{PHz})$ to $400 \mathrm{~nm}$ (750 THz). The electromagnetic spectrum of light is shown in Figure 2.

Optical properties determine the response of materials to incident electromagnetic radiation. The optical properties of a material are influenced by the nature of its surface and its electronic structure. The most known properties are reflection, refraction, transmission, and absorption. For each material, the incident radiation is partially transmitted, partially reflected, and partially absorbed.

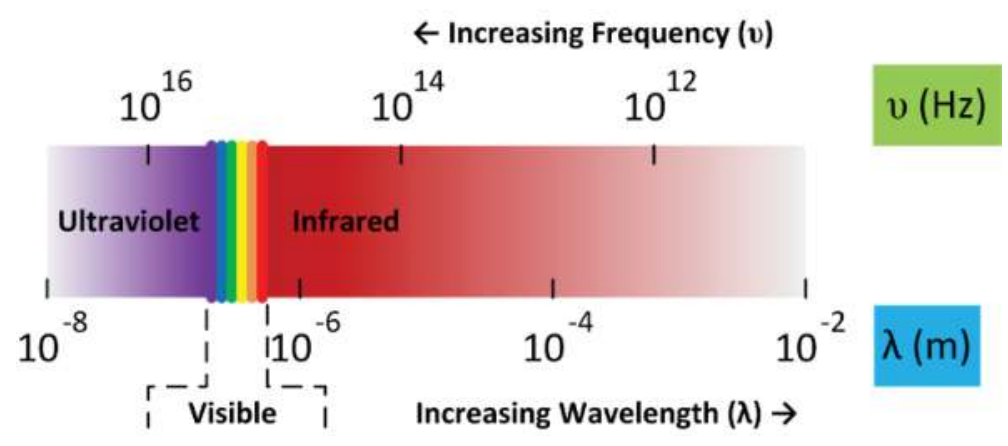

Figure 2. The electromagnetic spectrum of light. 
When light passes through a medium, some part of it will always be attenuated. This can be conveniently considered by defining a complex refractive index $n_{c}=n+j \kappa$, where the real part $(n)$ is the refractive index and indicates the phase velocity, whereas the imaginary part $(\kappa)$ is called the extinction coefficient or mass attenuation coefficient and it indicates the amount of attenuation when the electromagnetic wave propagates through the material.

The electric permittivity $\varepsilon$ and magnetic permeability $\mu$ of a medium determine the phase velocity $v=c / n$ of electromagnetic radiation through that medium. These are related by means of the relationship $\varepsilon \mu=1 / v^{2}$, where $c$ is the speed of light in vacuum and $n$ is the refractive index of the optical medium. The response of materials to alternating electric fields is characterized by a complex permittivity defined as $\varepsilon=\varepsilon^{\prime}+j \varepsilon^{\prime \prime}$, where $\varepsilon^{\prime}$ is the real part of the permittivity, which is related to the stored energy within the medium; and $\varepsilon^{\prime \prime}$ is the imaginary part of the permittivity, which is related to the dissipation (or loss) of energy within the medium. In addition, complex permittivity $\varepsilon$ must have poles only for positive imaginary parts, and therefore satisfies the Kramers-Kronig relations. The imaginary part of $\varepsilon$ leads to absorption loss if it is positive and absorption gain if it is negative.

Absorption coefficient, attenuation coefficient, or narrow beam attenuation coefficient of a material characterizes how easily it can be penetrated by a beam of light. A large attenuation coefficient means that the beam is quickly "attenuated" (weakened) as it passes through the medium, and a small attenuation coefficient means that the medium is relatively transparent to the beam. The SI unit of attenuation coefficient is the reciprocal meter $\left(\mathrm{m}^{-1}\right)$. A small attenuation coefficient suggests that the material is relatively transparent, while a larger value suggests greater degrees of opacity. The attenuation coefficient depends upon the type of material and the energy of the radiation. In addition, for electromagnetic radiation, the higher the energy of the incident light and the lower dense the material to be characterized, the lower the corresponding attenuation coefficient will be.

The chromatic dispersion of an optical medium is the phenomenon where the phase velocity and group velocity of light propagating in a transparent medium depend on the optical frequency. It is defined as $d n / d \lambda$. A related quantitative measure is the group velocity dispersion. The group index or group velocity refractive index $\left(n_{\mathrm{g}}\right)$ is defined as $n_{\mathrm{g}}=c / v_{\mathrm{g}}$ where $v_{\mathrm{g}}$ is the group velocity. When the dispersion is small, the group velocity can be linked to the phase velocity by the relationship $v_{\mathrm{g}}=v-\lambda(d v / d \lambda)$, where $\lambda$ is the wavelength in the medium. In this case, the group index can be written in terms of the wavelength dependence of the refractive index as $n_{\mathrm{g}}=n /(1+(\lambda / n)(d n / d \lambda))$.

A summary of the optical properties of the two-dimensional materials is provided in Table 1. These properties cannot be used individually to evaluate the optical performance of a material. The relative permittivity of the material can predict, for example, whether the material can be transparent or not by the sign and value of the real and imaginary parts, but it is not the only argument to conclude about this quality of the material. Relative permittivity of different two-dimensional materials used in nanophotonics and optoelectronics are shown in Figure 3.

The refractive index of photonic materials such as two-dimensional materials varies by the photon energy and/or wavelength of the light applied. Using the refractiveindex.info database, 


\begin{tabular}{|c|c|c|c|c|c|c|}
\hline Material & $\begin{array}{l}\text { Refractive } \\
\text { index }(n)\end{array}$ & $\begin{array}{l}\text { Extinction } \\
\text { coefficient }(\kappa)\end{array}$ & $\begin{array}{l}\text { Relative } \\
\text { permittivity }(\varepsilon)\end{array}$ & $\begin{array}{l}\text { Absorption } \\
\text { coefficient } \\
(\mu)\left(\mathrm{cm}^{-1}\right)\end{array}$ & $\begin{array}{l}\text { Chromatic } \\
\text { dispersion } \\
(d n / d \lambda)\left(\mu \mathrm{m}^{-1}\right)\end{array}$ & $\begin{array}{l}\text { Group } \\
\text { index }\left(n_{g}\right)\end{array}$ \\
\hline Graphene & 1.0575 & 2.2962 & $\begin{array}{l}\varepsilon^{\prime}=-4.1541 \\
\varepsilon^{\prime \prime}=4.8564\end{array}$ & 1.1621E06 & 25.520 & -5.2791 \\
\hline Borophene & 2.3465 & 1.5154 & $\begin{array}{l}\varepsilon^{\prime}=3.2098 \\
\varepsilon^{\prime \prime}=7.1119\end{array}$ & $1.0521 \mathrm{E} 06$ & 4.1925 & 1.5877 \\
\hline Indiene & 0.7000 & 4.7000 & $\begin{array}{l}\varepsilon^{\prime}=-21.600 \\
\varepsilon^{\prime \prime}=6.5800\end{array}$ & $1.0739 \mathrm{E} 06$ & 1.9000 & -0.3450 \\
\hline Stanene & 2.1600 & 6.3500 & $\begin{array}{l}\varepsilon^{\prime}=-35.657 \\
\varepsilon^{\prime \prime}=27.432\end{array}$ & $1.0931 \mathrm{E} 06$ & 3.1429 & -0.13429 \\
\hline Bismuthene & 4.0890 & 4.0890 & $\begin{array}{l}\varepsilon^{\prime}=-2.8729 \\
\varepsilon^{\prime \prime}=36.199\end{array}$ & $0.0525 \mathrm{E} 06$ & 5.2612 & -1.4879 \\
\hline InAs & 3.7973 & 0.49254 & $\begin{array}{l}\varepsilon^{\prime}=14.177 \\
\varepsilon^{\prime \prime}=3.7407\end{array}$ & $0.0848 \mathrm{E} 06$ & -0.94298 & 4.4857 \\
\hline $\mathrm{MoO}_{3}$ & 2.0576 & 0.59358 & $\begin{array}{l}\varepsilon^{\prime}=3.8813 \\
\varepsilon^{\prime \prime}=2.4427\end{array}$ & $0.3004 \mathrm{E} 06$ & -0.5988 & 2.2063 \\
\hline $\mathrm{MoS}_{2}$ & 2.3156 & 2.6594 & $\begin{array}{l}\varepsilon^{\prime}=-1.7101 \\
\varepsilon^{\prime \prime}=12.316\end{array}$ & $1.3459 \mathrm{E} 06$ & 18.621 & -2.3079 \\
\hline $\mathrm{MoSe}_{2}$ & 1.9136 & 2.3809 & $\begin{array}{l}\varepsilon^{\prime}=-2.0069 \\
\varepsilon^{\prime \prime}=9.1125\end{array}$ & $1.2050 \mathrm{E} 06$ & 9.7345 & -0.50345 \\
\hline $\mathrm{MoTe}_{2}$ & 1.8903 & 2.3354 & $\begin{array}{l}\varepsilon^{\prime}=-1.8809 \\
\varepsilon^{\prime \prime}=8.8292\end{array}$ & 1.1819E06 & 18.017 & -2.5834 \\
\hline
\end{tabular}

Table 1. Optical properties for a small set of two-dimensional materials.

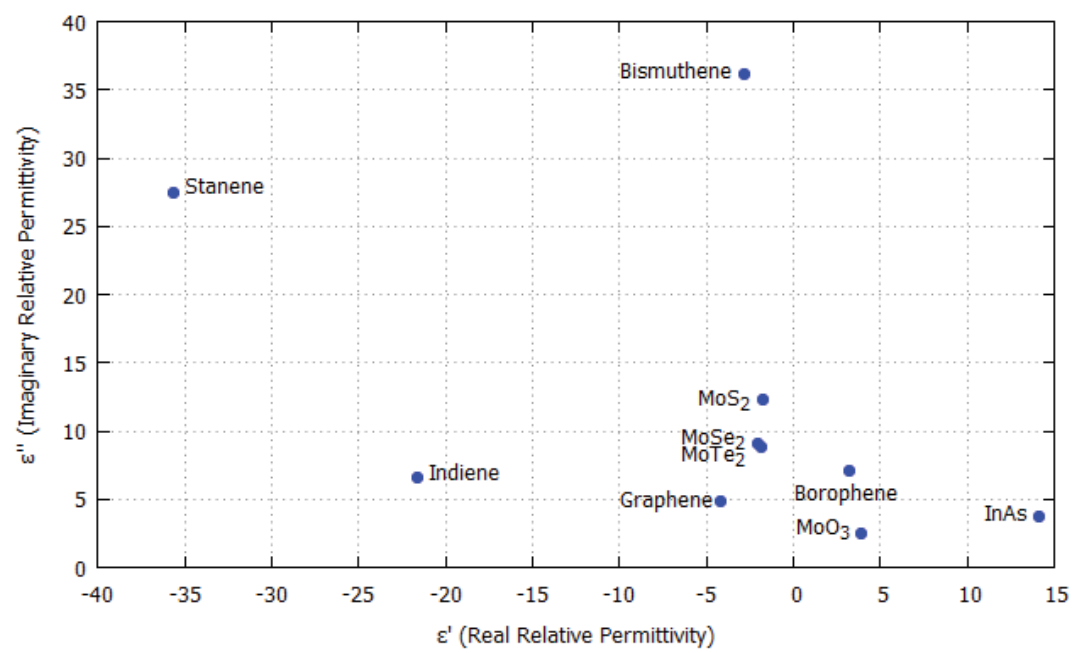

Figure 3. Relative permittivity of different two-dimensional materials. 
a pair of graphs has been obtained to know the performance of two-dimensional materials (see Figures 4 and 5). Materials with very high or very low refractive index do not have the greatest transparency necessarily, as visualized through the set of graphs obtained. A wide range of optical properties can be obtained through two-dimensional materials.
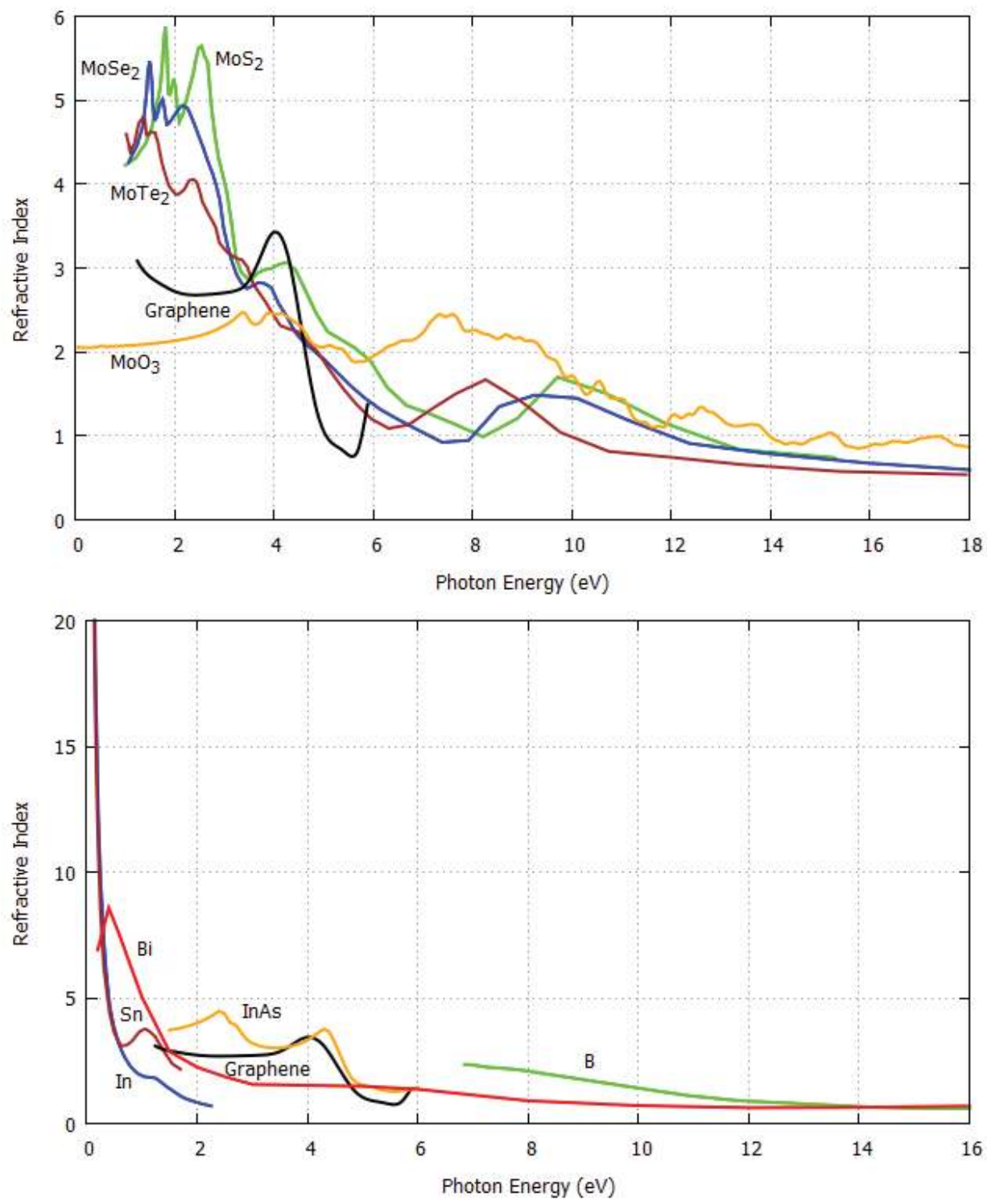

Figure 4. Refractive index versus photon energy for different two-dimensional materials. 

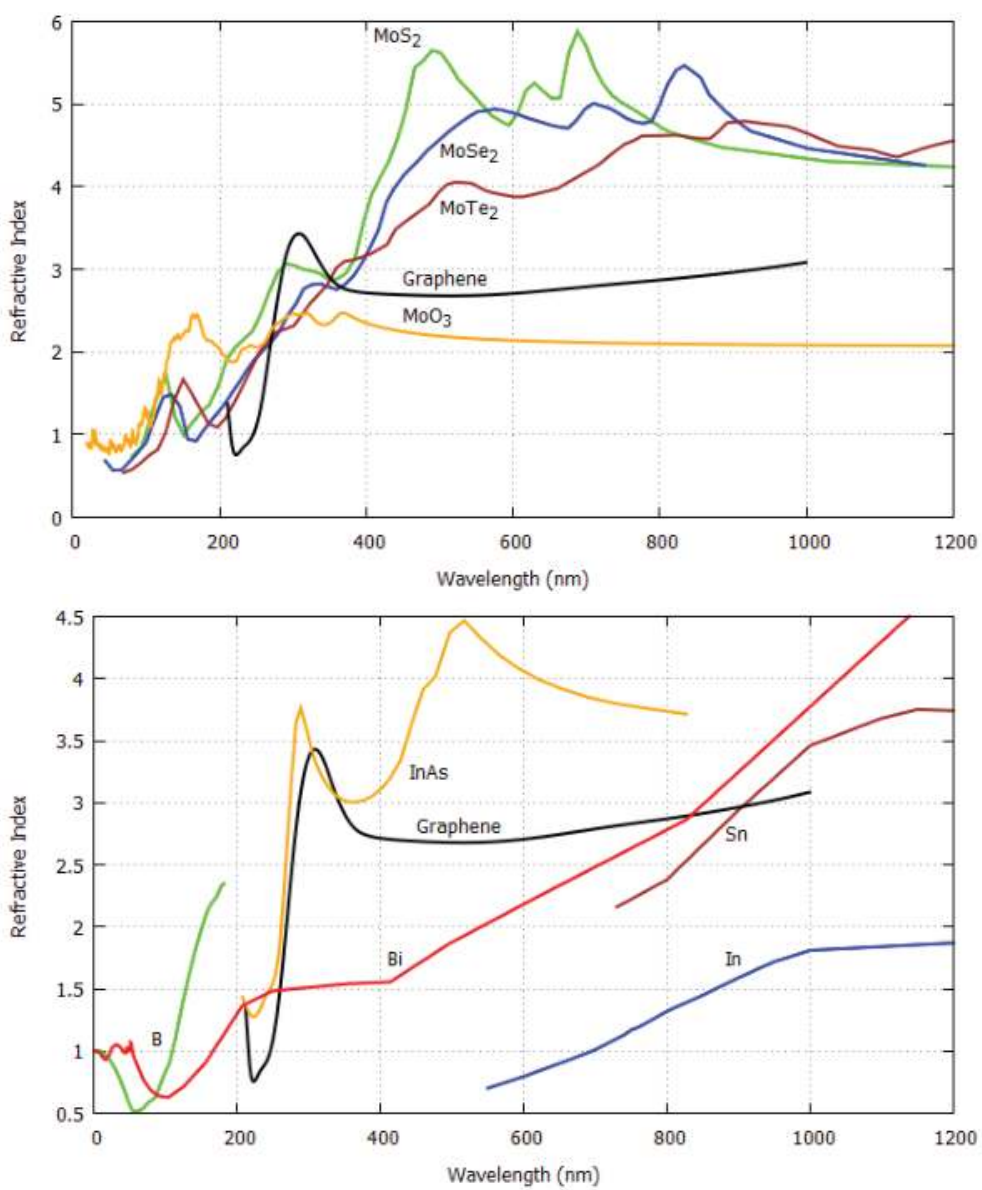

Figure 5. Refractive index versus wavelength for different two-dimensional materials.

Transmittance of the surface of a material is its effectiveness in transmitting radiant energy. It can be defined as the fraction of incident electromagnetic power that is transmitted through a material. Internal transmittance refers to energy loss by absorption, whereas total transmittance is due to absorption, scattering, reflection, etc. A set of transmittance graphs have been obtained through the refractiveindex.info database. These graphs are based on a spectral line or wavelength laser with value $0.2483 \mu \mathrm{m}(\mathrm{KrF})$ for different two-dimensional materials, and the results are outlined in Figures 6-15. The graphs contain the transmittance behavior for different thicknesses of two-dimensional materials ranging from 0.1 to $10 \mathrm{~nm}$. Indiene and stanene present low transmittance and other two-dimensional materials have high transmittance in all optical spectra, as shown in Figures 6-15. The value of the transmittance varies widely throughout the optical range, so the designer should check in which wavelength range his device or system must operate to properly choose the two-dimensional material will be used in its photonic or optoelectronic design. 


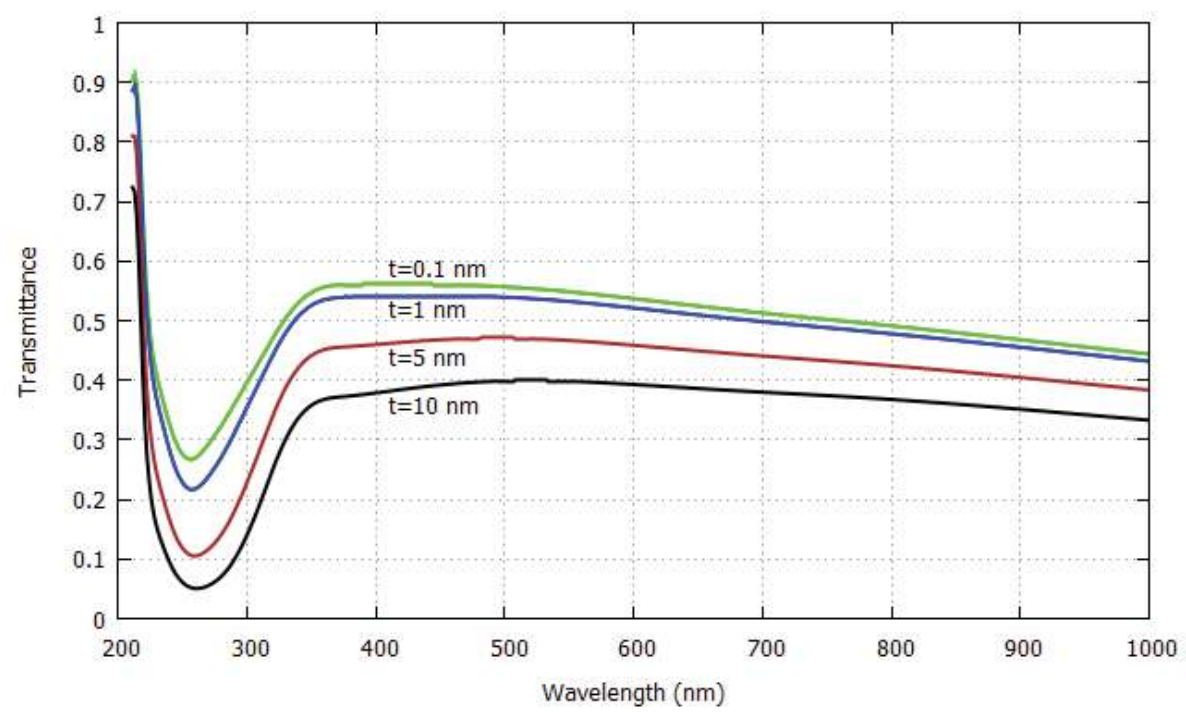

Figure 6. Total transmittance versus wavelength in graphene for different thicknesses.

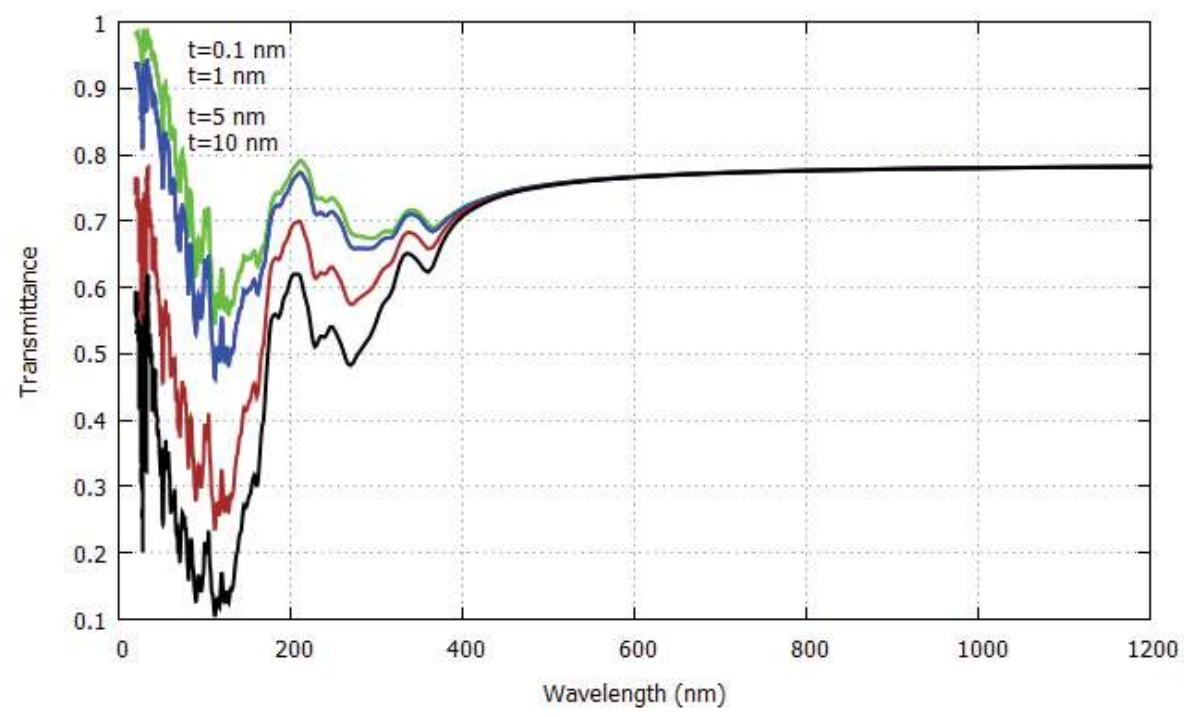

Figure 7. Total transmittance versus wavelength in tungsten(VI) oxide for different thicknesses.

After reviewing the different graphs obtained, it is possible to affirm that the two-dimensional materials can allow the development of emitting materials, waveguides, and light detectors throughout the optical spectrum. 


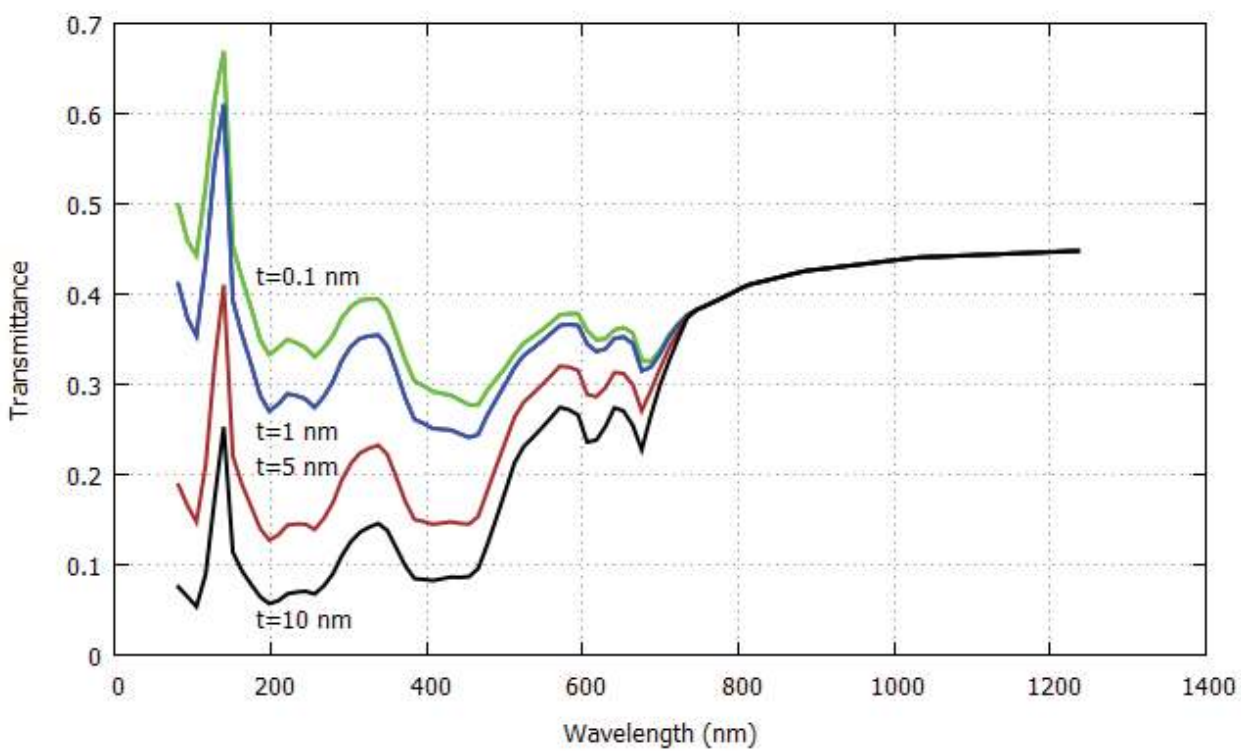

Figure 8. Total transmittance versus wavelength in molybdenum disulfide for different thicknesses.

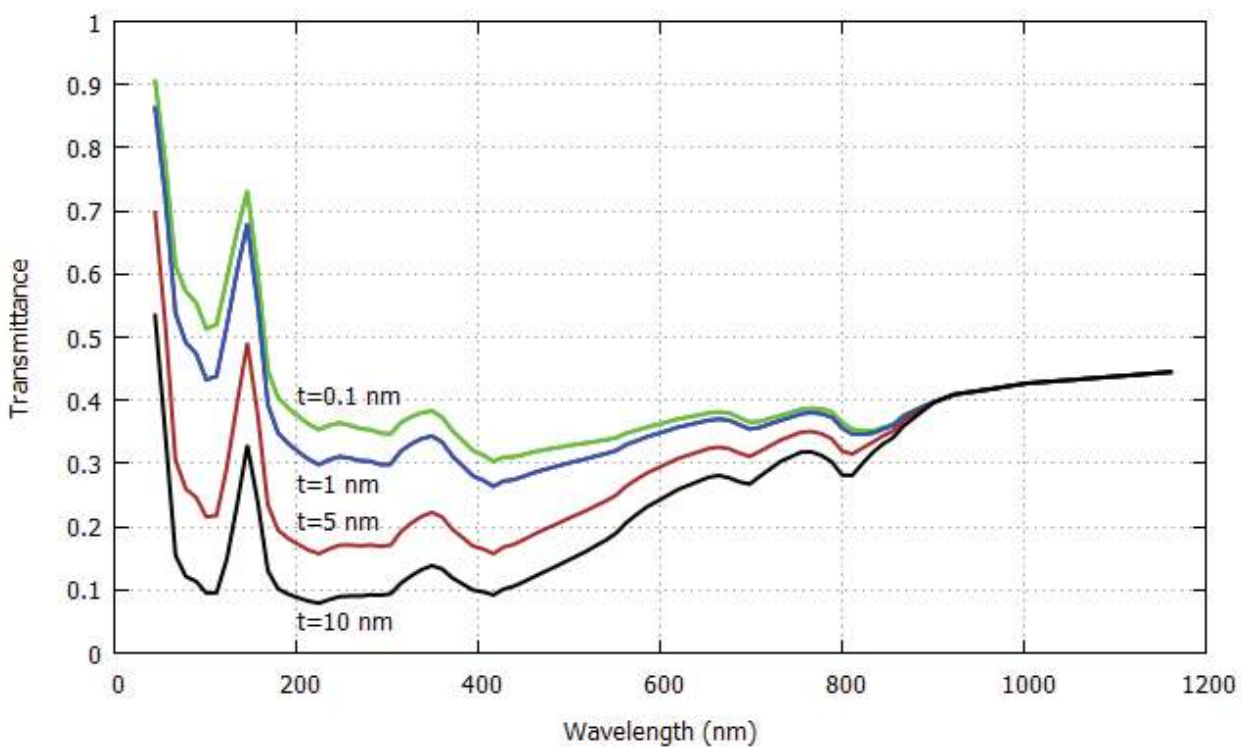

Figure 9. Total transmittance versus wavelength in molybdenum diselenide for different thicknesses. 


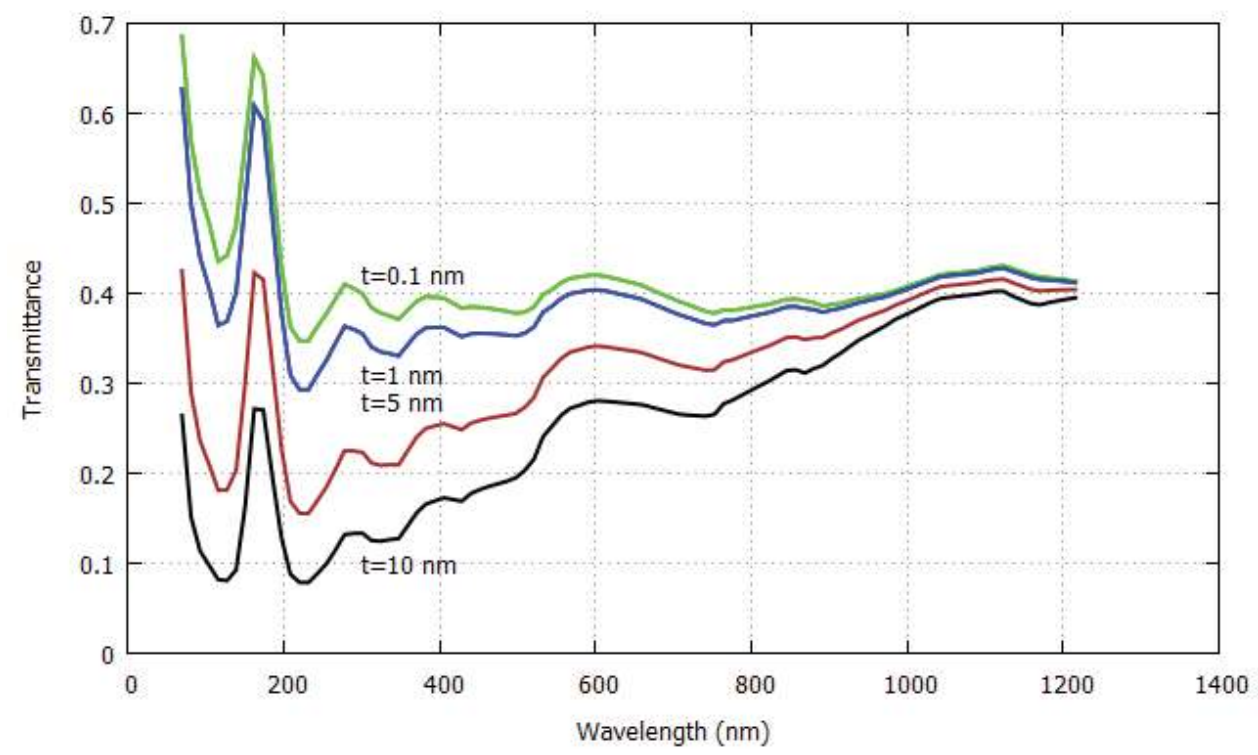

Figure 10. Total transmittance versus wavelength in molybdenum telluride for different thicknesses.

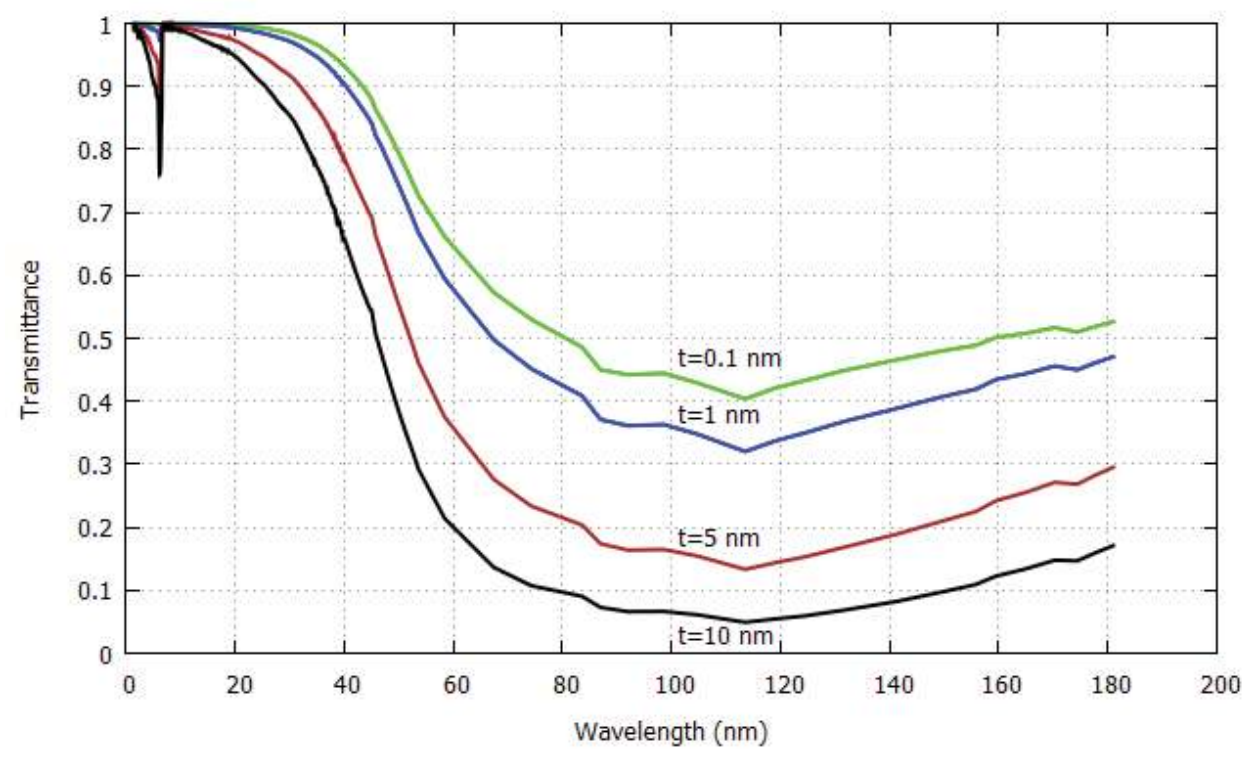

Figure 11. Total transmittance versus wavelength in borophene for different thicknesses. 


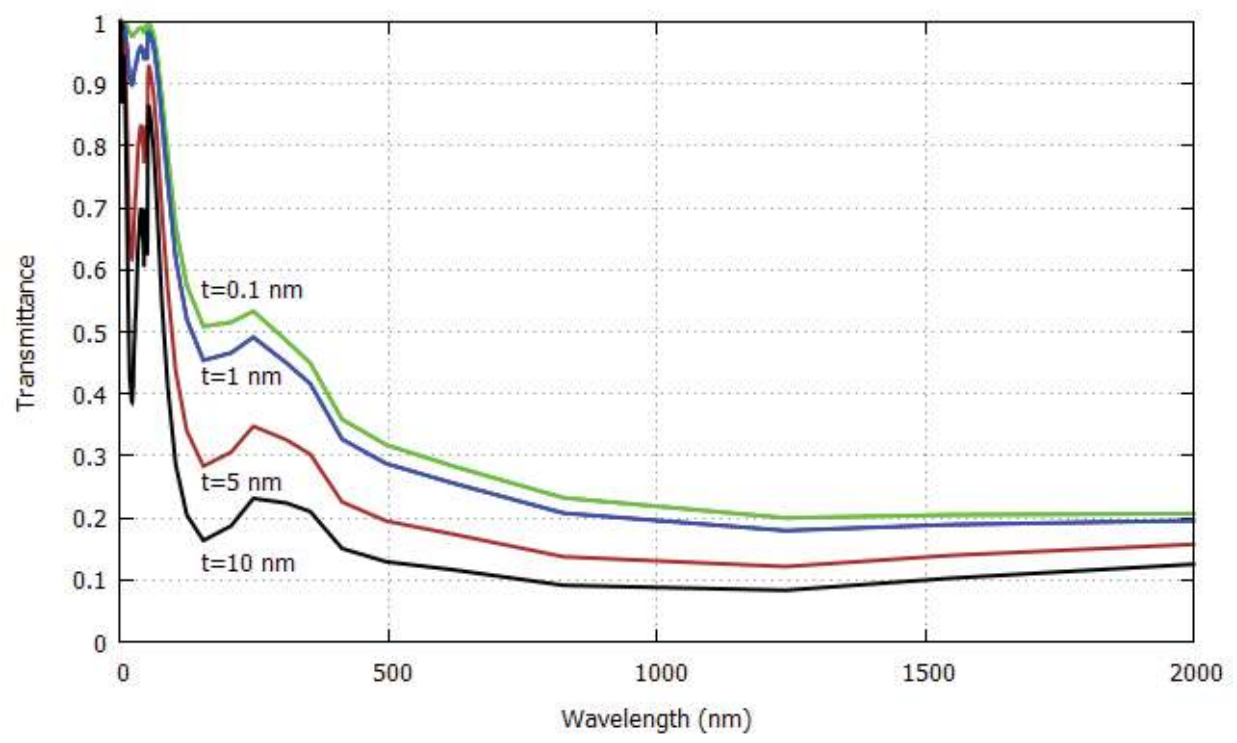

Figure 12. Total transmittance versus wavelength in bismuthene for different thicknesses.

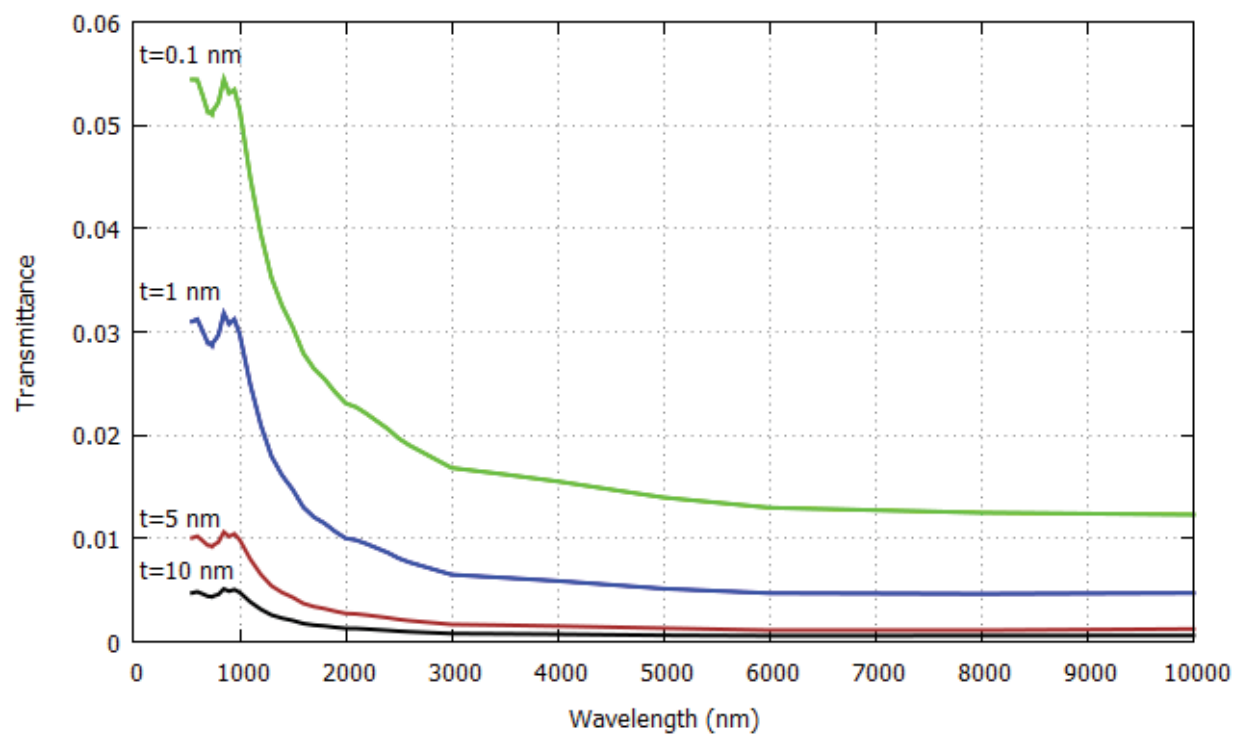

Figure 13. Total transmittance versus wavelength in indiene for different thicknesses. 


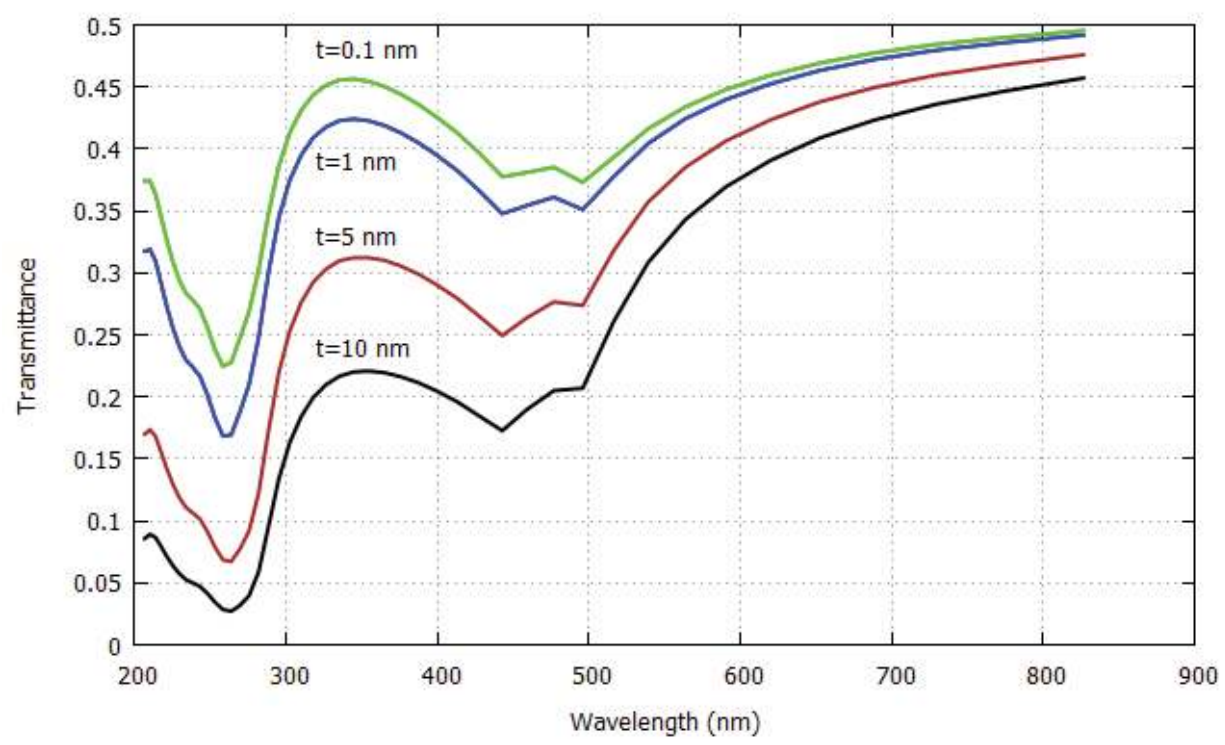

Figure 14. Total transmittance versus wavelength in arsenide for different thicknesses.

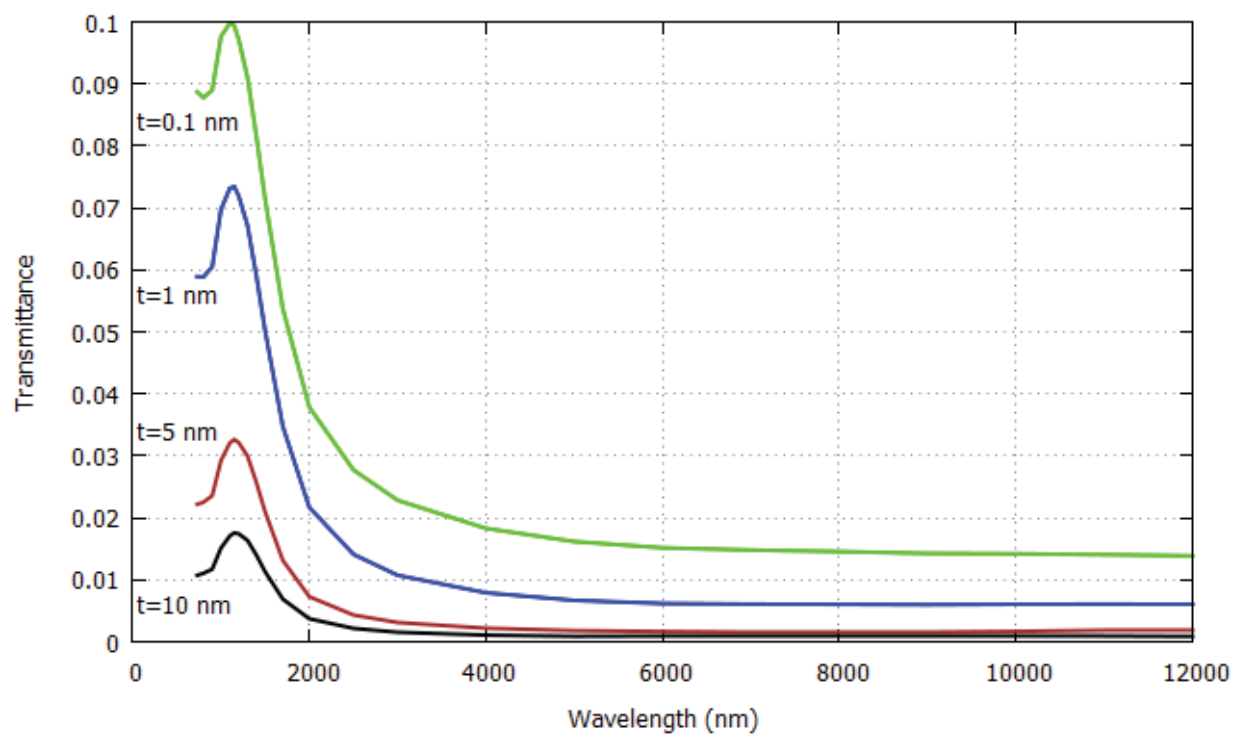

Figure 15. Total transmittance versus wavelength in stanene for different thicknesses. 


\section{Applications of the two-dimensional materials in photonics and optoelectronics}

Two-dimensional materials due to their semiconductor behavior with direct band gap allow the development of numerous optoelectronic applications thanks to the access to spin and valley degrees of freedom. Among the main photonic applications that can be developed using two-dimensional materials are light-emitting diodes, laser diodes, photoluminescent materials, photodetectors, waveguides, optical modulators, and in valleytronics.

\subsection{Light-emitting diodes}

A light-emitting diode can be defined as a two-terminal semiconductor light source, which is based in a $p$ - $n$ junction diode that emits light when it is activated by means of an electric field [2]. When an electrical voltage is applied to the terminals, electrons can recombine with holes within the device, releasing light in the form of photons. This effect is called electroluminescence, and the color of the light (visible wavelength) is determined by the energy band gap of the semiconductor material used in the manufacture of the emitter. In addition, these emitters are available for ultraviolet, visible, and infrared wavelengths.

The typical two-dimensional semiconductors, such as $\mathrm{MoS}_{2}, \mathrm{MoSe}_{2^{\prime}}, \mathrm{WS}_{2}, \mathrm{WSe}_{2}$, and phosphorene, have band gaps that are smaller than $2.0 \mathrm{eV}$ reducing their photoresponse in the blue and UV range $[7,8]$. Arsenene and antimonene present wide band gaps and high stability since they have an indirect bandgap as two-dimensional monolayers, and these under small biaxial strain can transform their electronic behavior from indirect into direct bang gap semiconductors. The values of refractive index are $2.3(\alpha-\mathrm{Sb})$ and $1.5(\beta-\mathrm{Sb})$ at the zero energy limit and scale up to 3.6 in the ultraviolet region [8].

The electroluminescence has been observed in two-dimensional monolayer field-effect transistors or $p$ - $n$ light-emitting diodes made of $\mathrm{MoS}_{2}[9,10]$, WSe ${ }_{2}[11]$, and $\mathrm{WS}_{2}$ [12]. Single layer molybdenum disulfide $\left(\mathrm{MoS}_{2}\right)$ field-effect transistors can emit electroluminescence (visible light) at $1.8 \mathrm{eV}$ thanks to its direct band gap [9]. Electrostatic gating must be used to improve control and efficiency of light emission. A light-emitting diode based on a two-dimensional monolayer $\mathrm{MoS}_{2}$ deposited on a heavily $p$-type doped silicon substrate [10]. This device emits a high energy exciton peak in $2.255 \mathrm{eV}$ through of a direct-exciton transition at room temperature which opened the possibility of controlling valley and spin excitation. The optimization of lighting, displays, optical interconnects, and optical sensors depends on the development of light-emitting diodes with high efficiency, well-defined spectral properties, compactness, and integrability [11]. The use of a thin boron nitride ( $h$-BN) support as a dielectric layer in a $p$ - $n$ light-emitting diode (LED) with a monolayer $\mathrm{WSe}_{2}$-induced electrostatically yields bright electroluminescence with 1000 times smaller injection current and 10 times smaller linewidth than in $\mathrm{MoS}_{2}$ monolayers. This quality can be obtained thanks to the control of the voltage applied to the LED. Ambipolar ionic liquid gated field-effect transistors based on $\mathrm{WS}_{2}$ monoand bilayers have electroluminescence at 2.14 and $1.82 \mathrm{eV}$, respectively [12]. It was observed that a much broader range of carrier density is achieved. 


\subsection{Laser diodes}

A laser diode can be defined as an electrically pumped semiconductor laser where active laser medium is formed by a $p-n$ junction of a semiconductor diode [2]. These devices regularly operate as directional lighting sources. These devices emit coherent light and they are supplied either by a forward electrical bias or an optical pumping. Optically pumped semiconductor lasers use a semiconductor material as the gain medium, or other laser (often another diode laser) as the pump source. This type allows a better wavelength selection and does not present interference from internal electrode structures.

A continuous-wave nanolaser can operate in the visible regime using a monolayer based on a two-dimensional material as a gain medium with the aim of confining the direct band excitons within one nanometer of the surface of a photonic crystal cavity (PCC) [13]. An optical pumping threshold, as low as $27 \mathrm{nW}$ at $130 \mathrm{~K}$, must be used to stimulate the spontaneous emission of photons. The use of surface-gain geometry allows an unprecedented accessibility, and thus the ability to tailor gain properties by means of external controls such as electrostatic gating and current injection, and therefore it can be operated using an electrical pumping. This topology is scalable and compatible with integrated photonics for on-chip communication technologies.

Advances in two-dimensional materials have allowed the implementation of a two-dimensional excitonic laser, by embedding a $\mathrm{WS}_{2}$ monolayer in a microdisk resonator, with a high-quality factor and optical confinement presenting a bright light at visible wavelengths [14]. This laser device has high quantum yield, small footprint, and low power consumption. This device allows the development of two-dimensional on-chip optoelectronics for high-performance optical communication and computing applications.

\subsection{Photoluminescence}

Photoluminescence can be defined as light emission from any form of matter after the absorption of photons [2]. The quality of tuning the optical properties of a material is important for the development of good photonic devices [1]. Two-dimensional materials present photoluminescence, which is defined as the light emission from any form of matter after the absorption of photons. Two-dimensional materials, such as graphene [15, 16], $\operatorname{MoS}_{2}[17-19], \operatorname{MoTe}_{2}[20,21]$, $\mathrm{WS}_{2}$ [22], $\mathrm{MoS}_{2}-\mathrm{WS}_{2}$ [23], $\mathrm{MoSe}_{2}$ [24, 25], and phosphorene [26], have been researched as photoluminescent materials. Luminescence can be achieved thanks to the reduction of thickness of materials, since it produces a transition from indirect band gap to a direct band gap. The doping or defects as well as strain associated with the presence of the boundaries in two-dimensional materials lead to strong photoluminescence quenching or enhancement [1]. Therefore, photoluminescence properties of two-dimensional materials can be tuned through chemical doping when dopants cover its surface [27]. Pressure produces a shift in the band gap energy of the material and therefore widens its spectrum although it reduces the photoluminescence intensity [28]. Uniaxial tensile strain increases the photoluminescence peak intensity in a twodimensional material, due to that it guarantees the direct band gap [29]. Electrical gating of two-dimensional materials increases photoluminescence intensity, whereas the photoluminescence wavelength remains nearly constant [30]. The photoluminescence of a two-dimensional 
material is strongly affected by the type of substrate where it is deposited [31]. Piezoelectric substrates such as $\mathrm{SrTiO}_{3}$ have a higher emission intensity than dielectric substrates such as $\mathrm{SiO}_{2}$ which reduces the emission intensity. In addition, the photoluminescence intensity from transition metal dichalcogenide alloys is drastically increased for monolayer samples composed of $\mathrm{MoS}_{2}-\mathrm{MoSe}_{2}$ and $\mathrm{MoS}_{2}-\mathrm{WS}_{2}$ with respect to multilayer samples [32].

Monolayer phosphorene operates as an exciton and trion emitter whose performance is comparable with other 2D transition metal dichalcogenide (TMD) semiconductors such as $\mathrm{MoS}_{2^{\prime}}$ although semiconductors such as $\mathrm{WSe}_{2}$ and $\mathrm{MoSe}_{2}$ present higher carrier lifetimes as shown in Figure 16 [33]. An exciton is the main mechanism for light emission in semiconductors at low temperature. The existence of exciton states may be inferred from the absorption of light associated with their excitation. Typically, excitons are observed just below the band gap. A trion is a localized exciton which consists of three charged quasiparticles. A negative trion consists of two electrons and one hole and a positive trion consists of two holes and one electron. Trion states were predicted theoretically and then observed experimentally in various optically excited semiconductors, especially in quantum dots and quantum well structures. The strong anisotropic atomic structure of single-layer black phosphorus makes that optical response be sensitive to the magnitude and the orientation of the applied strain to the material [34]. Biaxial strain can tune the optical band gap of phosphorene from $0.38 \mathrm{eV}$ (at $-8 \%$ strain) to $2.07 \mathrm{eV}$ (at $5.5 \%)$.

\subsection{Photodetectors}

A photodetector or photosensor is an electronic device that converts the photons of light into electrical current [2]. The operating principle of a photodetector can be basically described as

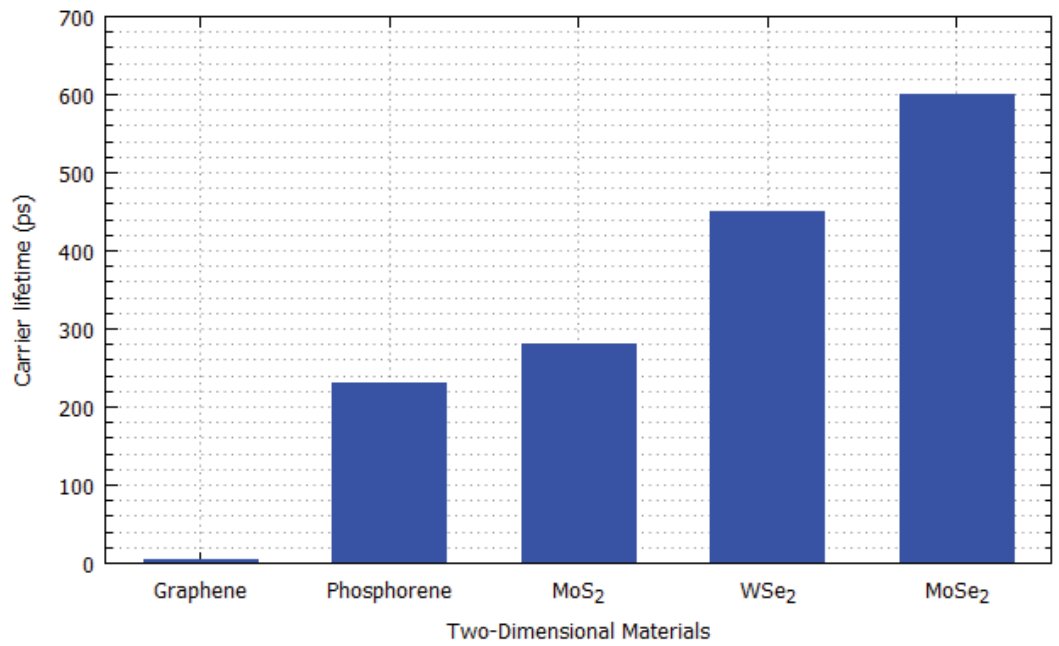

Figure 16. Carrier lifetimes in different two-dimensional materials. 
follows. When the photons of light incident on a semiconductor material have energy greater than the forbidden band of this material, they create electron-hole pairs (excitons) or free carriers depend on the exciton binding energy in the material. Bound excitons separated by an applied or built-in electric field can generate a photocurrent. Photodetectors based on semiconductor materials can be classified into two large groups: photodiodes and phototransistors. The former has a much faster response and the latter have better sensitivities.

A graphene photodetector working in the photoconductor mode and based on photothermoelectric effect and bolometric effect (zero and nonzero biased operations, respectively) was integrated on silicon nitride $\left(\mathrm{Si}_{3} \mathrm{~N}_{4}\right)$ waveguide [35]. This combination of materials facilitates unprecedented nonlinear and optoelectronic applications. The graphene has a behavior as a highly $p$-doped semiconductor that produces a photocurrent generation.

The ultrashort lifetime of photocarriers, caused by the fast recombination of graphene, results in the weak response of light and limits its application in photodetection [36]. With the aim of improving the performance of the graphene in photodetection, it must be coupled with photonic structures or building vertical heterostructures by stacking graphene with other layered materials and increasing the doping level considering the band slop and the absorption at certain wavelengths.

Monolayer $\mathrm{WSe}_{2}$ and $\mathrm{MoS}_{2}$ heterostructures present strong photoluminescence intensity, which suggest that there is a strong interlayer coupling of the charge carriers [5].

\subsection{Solar cells}

A solar cell or photovoltaic cell can be defined as an electrical device that converts the energy of light directly into electricity by the photovoltaic effect, that is, a physical and chemical phenomenon where light is absorbed causing excitation of an electron or other charge carrier to a higher energy state [2]. Electrical properties, such as current, voltage, or resistance, vary when solar cells are exposed to light.

Thanks to their semiconducting character, graphene and monolayer transition metal dichalcogenides can be considered as potential sunlight absorbers in thicknesses less than $1 \mathrm{~nm}$, which represents a value with 1 order of magnitude higher than for GaAs and $\mathrm{Si}$ [37]. Two stacked monolayers have been proposed: (1) a Schottky barrier solar cell between $\mathrm{MoS}_{2}$ and graphene and (2) an excitonic solar cell based on a $\mathrm{MoS}_{2} / \mathrm{WS}_{2}$ bilayer. These structures can reach power conversion efficiencies of up to $\sim 1 \%$, corresponding to values of approximately 1 to 3 orders of magnitude higher than to power densities of the best existing ultrathin solar cells.

The ultrathin transition metal dichalcogenide films and their Van der Waals heterostructures have been used to develop photovoltaic devices with the aim of offering superior performance, extreme flexibility, and long lifetime due to suitable band gap and excellent light absorption capability [38]. A $p-\mathrm{Si} / \mathrm{MoS}_{2}$ heterojunction-based solar cell produces an enormous quantity of photogenerated carriers and increases the photocurrent generated by them. The semiconductor heterostructures and superlattices represent a solar cell design platform without the limitation of lattice matching present in other materials [5]. 


\subsection{Waveguides}

An optical waveguide can be defined as a physical structure that guides or transports electromagnetic waves in the optical spectrum [2]. The most common types of optical waveguides are optical fibers and rectangular waveguides. These are commonly used as components in integrated optical circuits or as a transmission medium in optical communication systems. In addition, waveguides can be classified by their geometry (planar, strips, fiber waveguides), mode structure (single-mode, multiple-mode), refractive index distribution (step index, gradient index), and material (glass, polymer, semiconductor material).

The ultrashort optical pulses can be propagated through graphene-based nanoribbon waveguides in telecommunications [39]. The pulses can experiment attenuation, high-order dispersive effects and nonlinear effects when they pass through graphene. Fortunately, these effects can be controlled with the input signal power and the chemical potential of the graphene. These waveguides can be applied to other nanophotonic devices such as filters, modulators, antennas, switches, and other devices.

\subsection{Optical modulators}

An optical modulator can be defined as a device used to modulate a beam of light, which is carried over free space or propagated through an optical waveguide [2]. These devices can be classified by the parameter of a light beam that is manipulated into amplitude modulators, phase modulators, polarization modulators, etc. The easiest way to perform the modulation of light intensity is to modulate the electric current that drives the light source, as in a laser diode. In addition, optical modulators can be classified by the properties of the materials used to modulate the light beam into two groups: absorptive modulators and refractive modulators. In absorptive modulators, the absorption coefficient of the material is changed; and in refractive modulators, the refractive index of the material is changed.

Two-dimensional materials can realize various functions at once due to their diverse physical properties as it is the case of the multifunctional optical modulators where optical modulators and photodetectors or optical modulators and plasmon waveguides can be implemented with the same semiconductor material [40]. Main advantages that these materials have on optical modulators are a broad operation bandwidth from the visible to terahertz range, small footprint, low cost, easy integration, and large flexibility. This quality facilitates the integration of diverse electronic and optical components with an "all-in-one" solution. However, before of reaching the commercial success of the two-dimensional materials, a trade-off between performance and cost must be considered.

\subsection{Valleytronics}

Particularly, transition metal dichalcogenide monolayers present a strong spin-orbit coupling, which leads to a spin-orbit splitting of hundreds meV in the valence band and a few $\mathrm{meV}$ in the conduction band $[1,41]$. This quality allows the control of the electron spin by tuning the excitation laser photon energy that is applied to the material. This quality allows 
controlling the electrical charge or spin degree of free carriers, and therefore, novel devices can be proposed. Among these devices, a WSe 2 -based electrically switchable chiral lightemitting transistor has been designed [42]. This device operates as a spin LED that shows circularly polarized electroluminescence, but depends on the application of an external magnetic field to operate. Circular polarized light can be used on three-dimensional displays or as spin sources for spintronics and as information carriers in quantum computation.

\section{Conclusions}

A lot of promising applications in nanophotonics will be developed in the coming decades. The main challenge for large-scale applications of two-dimensional materials is the ability to control the synthesis processes in order to optimize the physical and chemical properties and to extend its use in a wide variety of novel photonic applications. Most applications in nanophotonics of two-dimensional materials are still in the research phase or stage of technological development. However, the progress reported in this work indicates that the practical implementation of devices and systems will be based on simple and heterogeneous structures. The main applications are focused on the development of photodetectors based on the principle of photoconductivity, transparent electrodes, waveguides, and solar cells based on the photoelectric effect. The large variety of available two-dimensional materials, their heterostructures and hybrid systems that are being researched will continue introducing novel properties to outperform competing technologies applied in electronics and photonics. Designers can make use of an innumerable amount of two-dimensional materials based on the different chemical elements that have been synthesized or designed in computer. Fortunately, graphene and its derivatives are no longer alone and several material choices, such as $\mathrm{MoS}_{2^{\prime}} \mathrm{MoSe}_{2^{\prime}} \mathrm{WS}_{2^{\prime}} \mathrm{WSe}_{2^{\prime}}$ phosphorene, borophene, arsenene, antimonene, bismuthene, indiene, and stanene, can be exploited through the optical range.

\section{Acknowledgements}

The author wants to thank financial support from CONACYT (contract no. 152524, basic science), Tecnológico Nacional de México (contract no. M00.1/1671/2015), and Instituto Tecnológico Superior de Irapuato (ITESI).

\section{Author details}

\section{Rafael Vargas-Bernal}

Address all correspondence to: ravargas@itesi.edu.mx

Department of Materials Engineering, Higher Technological Institute of Irapuato, Irapuato, Guanajuato, Mexico 


\section{References}

[1] Kolobov, A.V., Tominaga, J. Two-Dimensional Transition-Metal Dichalcogenides. Switzerland: Springer; 2016. 545 p. DOI: 10.1007/978-3-319-31450-1

[2] Liu, J.-M. Principles of Photonics. Cambridge, United Kingdom: Cambridge University Press; 2016. 260 p. DOI: 10.1017/CBO9781316687109

[3] Ponraj, J.S., Xu, Z.-Q., Dhanabalan, S.C., Mu, H., Wang, Y., Yuan, J., Li, P., Thakur, S., Ashrafi, M., Mccoubrey, K. Photonics and Optoelectronics of Two-Dimensional Materials beyond Graphene. Nanotechnology. 2016; 27(46): 462001. DOI: 10.1088/0957-4484/27/46/462001

[4] Xia, F., Wang, H., Xiao, D., Dubey, M., Ramasubramaniam A. Two-Dimensional Material Nanophotonics. Nature Photonics. 2014; 8(12): 899-907. DOI: 10.1038/nphoton.2014.271

[5] Duan, X., Wang, C., Pan, A., Yu, R., Duan, X. Two-Dimensional Transition Metal Dichalcogenides as Atomically Thin Semiconductors: Opportunities and Challenges. Chemical Society Reviews. 2015; 44(24): 8859-8876. DOI: 10.1039/c5cs00507h

[6] Tian, H., Chin, M.L., Najmaei, S., Guo, Q., Xia, F., Wang, H., Dubey, M. Optoelectronic Devices based on Two-Dimensional Transition Metal Dichalcogenides. Nano Research. 2016; 9(6): 1543-1560. DOI: 10.1007/s12274-016-1034-9

[7] Zhang, S., Yan, Z., Li, Y., Chen, Z., Zeng, H. Atomically Thin Arsenene and Antimonene: Semimetal-Semiconductor and Indirect-Direct Band-Gap Transitions. Angewandte Chemie International Edition. 2015; 54(10): 3112-3115. DOI: 10.1002/anie.201411246

[8] Singh, D., Gupta, S.K., Sonvane, Y., Lukačević, I. Antimonene: A Monolayer Material for Ultraviolet Optical Nanodevices. Journal of Materials Chemistry C. 2016; 4(26): 63866390. DOI: 10.1039/c6tc01913g

[9] Krane, N., Lotze, C., Läger, J.M., Reecht, G., Franke, K.J. Electronic Structure and Luminescence of Quasi-Freestanding $\mathrm{MoS}_{2}$ Nanopatches on $\mathrm{Au}(111)$. Nano Letters. 2016; 16(8): 5163-5168. DOI: 10.1021/acs.nanolett.6b02101

[10] Ye, Y., Ye, Z., Gharghi, M., Zhu, H., Zhao, M., Wang, Y., Yin, X., Zhang, X. Exciton-dominant Electroluminescence from a Diode of Monolayer $\mathrm{MoS}_{2}$. Applied Physics Letters. 2014; 104(19): 193508. DOI: 10.1063/1.4875959

[11] Ross, J.S., Klement, P., Jones, A.M., Ghimire, N.J., Yan, J., Mandrus, D.G., Taniguchi, T., Watanabe, K., Kitamura, K., Yao, W., Cobden, D.H., Xu, X. Electrically Tunable Excitonic Light-Emitting Diodes based on Monolayer WSe ${ }_{2}$ p-n Junctions. Nature Nanotechnology. 2014; 9(4): 268-272. DOI: 10.1038/nnano.2014.26

[12] Jo, S., Ubrig, N., Berger, H., Kuzmenko, A.B., Morpurgo, A.F. Mono- and Bilayer WS Light-Emitting Transistors. Nano Letters. 2014; 14(4): 2019-2025. DOI: 10.1021/nl500171v

[13] Wu, S., Buckley, S., Schaibley, J.R., Feng, L., Yan, J., Mandrus, D.G., Hatami, F., Yao, W., Vučković, J., Majumdar, A., Xu, X. Monolayer Semiconductor Nanocavity Lasers with Ultralow Thresholds. Nature. 2015; 520(7545): 69-72. DOI: 10.1038/nature14290 
[14] Ye, Y., Wong, Z.J., Lu, X., Ni, X., Zhu, H., Chen, X., Wang, Y., Zhang, X. Monolayer Excitonic Laser. Nature Photonics. 2015; 9(11): 733-737. DOI: 10.1038/nphoton.2015.197

[15] Pal, S.K. Versatile Photoluminescence from Graphene and Its Derivatives. Carbon. 2015; 88: 86-112. DOI: 10.1016/j.carbon.2015.02.035

[16] Du, D., Song, H., Nie, Y., Sun, X., Chen, L., Ouyang, J. Photoluminescence of Graphene Oxide in Visible Range Arising from Excimer Formation. Journal of Physical Chemistry C. 2015; 119(34): 20085-20090. DOI: 10.1021/acs.jpcc.5b04529

[17] Gao, W., Lee, Y.H., Jiang, R., Wang, J., Liu, T., Ling, X.Y. Localized and Continuous Tuning of Monolayer $\mathrm{MoS}_{2}$ Photoluminescence Using a Single Shape-Controlled Ag Nanoantenna. Advanced Materials. 2016; 28(4): 701-706. DOI: 10.1002/adma.201503905

[18] Fan, X., Xu, P., Li, Y.C., Zhou, D., Sun, Y., Nguyen, M.A.T., Terrones, M., Mallouk, T.E. Controlled Exfoliation of $\mathrm{MoS}_{2}$ Crystals into Trilayer Nanosheets. Journal of the American Chemical Society. 2016; 138(15): 5143-5149. DOI: 10.1021/jacs.6b01502

[19] Andleeb, S., Singh, A.K., Eom, J. Chemical Doping of $\mathrm{MoS}_{2}$ Multilayer by p-Toluene Sulfonic Acid. Science and Technology of Advanced Materials. 2015; 16(3): 035009. DOI: 10.1088/1468-6996/16/3/035009

[20] Lezama, I.G., Arora, A., Ubaldini, A., Barreteau, C., Giannini, E., Potemski, M., Morpurgo, A.F. Indirect-to-Direct Band Gap Crossover in Few-Layer MoTe ${ }_{2}$ Nano Letters. 2015; 15(4): 2336-2342. DOI: 10.1021/nl5045007

[21] Ruppert, C., Aslan, O.B., Heinz, T.F. Optical Properties and Band Gap of Single- and Few-layer $\mathrm{MoTe}_{2}$ Crystals. Nano Letters. 2014; 14(11): 6231-6236. DOI: 10.1021/nl502557g

[22] McCreary, K.M., Hanbicki, A.T., Jernigan, G.G., Culbertson, J.C., Jonker, B.T. Synthesis of Large-Area WS 2 Monolayers with Exceptional Photoluminescence. Scientific Reports. 2016; 6: 19159. DOI: 10.1038/srep19159

[23] Chen, Y., Xi, J., Dumcenco, D.O., Liu, Z., Suenaga, K., Wang, D., Shuai, Z., Huang, Y-S., Xie, L. Tunable Band Gap Photoluminescence from Atomically Thin Transition-Metal Dichalcogenide Alloys. ACS Nano. 2013; 7(5): 4610-4616. DOI: 10.1021/nn401420h

[24] Kang, J., Zhang, L., Wei, S.-H. A Unified Understanding of the Thickness-dependent Bandgap Transition in Hexagonal Two-Dimensional Semiconductors. Journal of Physical Chemistry Letters. 2016; 7(4): 597-602. DOI: 10.1021/acs.jpclett.5b02687

[25] Wang, G., Palleau, E., Amand, T., Tongay, S., Marie, X., Urbaszek, B. Polarization and Time-resolved Photoluminescence Spectroscopy of Excitons in $\mathrm{MoSe}_{2}$ Monolayers. Applied Physics Letters. 2015; 106(11): 112101. DOI: 10.1063/1.4916089

[26] Zhang, S., Yang, J., Xu, R., Wang, F., Li, W., Ghufran, M., Zhang, Y.-W., Yu, Z., Zhang, G., Qin, Q., Lu, Y. Extraordinary Photoluminescence and Strong Temperature/Angledependent Raman Response in Few-Layer Phosphorene. ACS Nano. 2014; 8(9): 95909596. DOI: $10.1021 / \mathrm{nn} 503893 \mathrm{j}$ 
[27] Liu, X., Qu, D., Ryu, J., Ahmed, F., Yang, Z., Lee, D., Yoo, W.J. P-type Polar Transition of Chemically doped Multilayer $\mathrm{MoS}_{2}$ Transistor. Advanced Materials. 2016; 28(12): 23452351. DOI: 10.1002/adma.201505154

[28] Nayak, A.P., Pandey, T., Voiry, D., Liu, J., Moran, S.T., Sharma, A., Tan, C., Chen, C.-H., Li, L.-J., Chhowalla, M., Lin, J.-F., Singh, A.K., Akinwande, D. Pressure-Dependent Optical and Vibrational Properties of Monolayer Molybdenum Disulfide. Nano Letters. 2015; 15(1): 346-353. DOI: 10.1021/n15036397

[29] Wang, Y., Cong, C., Yang, W., Shang, J., Peimyoo, N., Chen, Y., Kang, J., Wang, J., Huang, W., Yu, T. Strain-induced Direct-Indirect Bandgap Transition and Phonon Modulation in Monolayer WSe ${ }_{2}$. Nano Research. 2015; 8(8): 2562-2572. DOI: 10.1007/s12274-015-0762-6

[30] Oh, H.M., Jeong, H., Han, G.H., Kim, H., Kim, J.H., Lee, S.Y., Jeong, S.Y., Jeong, S., Park, D.J., Kim, K.K., Lee, Y.K., Jeong, M.S. Modulating Electronic Properties of Monolayer $\mathrm{MoS}_{2}$ via Electron-withdrawing Functional Groups of Graphene Oxide. ACS Nano. 2016, 10(11): 10446-10453. DOI: 10.1021/acsnano.6b06319

[31] Li, Y., Qi, Z., Liu, M., Wang, Y., Cheng, X., Zhang, G., Sheng, L. Photoluminescence of Monolayer $\mathrm{MoS}_{2}$ on $\mathrm{LaAlO}_{3}$ and $\mathrm{SrTiO}_{3}$ Substrates. Nanoscale. 2014; 6(24): 15248-15254. DOI: $10.1039 / \mathrm{c} 4 \mathrm{nr} 04602 \mathrm{a}$

[32] Choudhary, N., Park, J., Hwang, J.Y., Chung, H.-S., Dumas, K.H., Khondaker, S.I., Choi, W., Jung, Y. Centimeter Scale Patterned Growth of Vertically Stacked Few Layer only 2D $\mathrm{MoS}_{2} / \mathrm{WS}_{2}$ van der Waals Heterostructure. Scientific Reports. 2016; 6: 25456. DOI: 10.1038/srep25456

[33] Yang, J., Xu, R., Pei, J., Myint, Y.W., Wang, F., Wang, Z., Zhang, S., Yu, Z., Lu, Y. Optical Tuning of Exciton and Trion Emissions in Monolayer Phosphorene. Light: Science \& Applications. 2015; 4(7): e312. DOI: 10.1038/1sa.2015.85

[34] Zhang, W., Ye, C., Hong, L., Yang, Z., Zhou, R. Molecular Structure and Dynamics of Water on Pristine and Strained Phosphorene: Wetting and Diffusion at Nanoscale. Scientific Reports. 2016; 6: 38327. DOI: 10.1038/srep38327

[35] Wang, J., Cheng, Z., Chen, Z., Xu, J.-B., Tsang, H.K., Shu, C. Graphene Photodetector Integrated on Silicon Nitride Waveguide. Journal of Applied Physics. 2015; 117(14): 144504. DOI: $10.1063 / 1.4917378$

[36] Lin, L., Liao, L., Yin, J., Peng, H., Liu, Z. Building Graphene p-n Junctions for NextGeneration Photodetection. Nano Today. 2015; 10: 701-716. DOI: 10.1016/j.nantod.2015. 11.006

[37] Furchi, M.M., Zechmeister, A.A., Hoeller, F., Wachter, S., Pospischil, A., Mueller, T. Photovoltaics in Van der Waals Heterostructures. IEEE Journal of Selected Topics in Quantum Electronics. 2017; 23(1): 4100111. DOI: 10.1109/JSTQE.2016.2582318

[38] Pradhan, S.K., Xiao, B., Pradhan, A.K. Enhanced Photo-Response in p-Si/MoS 2 Heterojunction-based Solar Cells. Solar Energy Materials \& Solar Cells. 2016; 144: 117-127. DOI: 10.1016/j.solmat.2015.08.021 
[39] Lima, Jr. A.W., Mota, J.C.M., Sombra, A.S.B. Attenuation, Dispersion and Nonlinearity Effects in Graphene-based Waveguides. Beilstein Journal of Nanotechnology. 2015; 6: 1221-1228. DOI: 10.3762/bjnano.6.125

[40] Sun, Z., Martinez, A., Wang, F. Optical Modulators with 2D Layered Materials. Nature Photonics. 2016; 10(4): 227-238. DOI: 10.1038/nphoton.2016.15

[41] Xie, L., Cui, X. Manipulating Spin-Polarized Photocurrents in 2D Transition Metal Dichalcogenides. PNAS. 2016; 113(14): 3746-3750. DOI: 10.1073/pnas.1523012113

[42] Zhang, Y.J., Oka, T., Ye, J.T., Iwasa, Y. Electrically Switchable Chiral Light-Emitting Transistor. Science. 2014; 344(6185): 725-728. DOI: 10.1126/science.1251329 
